\title{
Evaluation of macrolides for possible use against multidrug-resistant Mycobacterium tuberculosis
}

\author{
Anne-Fleur van der Paardt ${ }^{1}$, Bob Wilffert ${ }^{1,2}$, Onno W. Akkerman ${ }^{3,4}$, \\ Wiel C.M. de Lange $e^{3,4}$, Dick van Soolingen ${ }^{5,6}$, Bhanu Sinha ${ }^{7}$, Tjip S. van der Werf ${ }^{4,8}$, \\ Jos G.W. Kosterink ${ }^{1,2}$ and Jan-Willem C. Alffenaar ${ }^{1}$
}

\begin{abstract}
Affiliations: ${ }^{1}$ University of Groningen, University Medical Center Groningen, Dept of Clinical Pharmacy and Pharmacology, Groningen, The Netherlands. ${ }^{2}$ University of Groningen, Dept of Pharmacy, Section Pharmacotherapy and Pharmaceutical Care, Groningen, The Netherlands. ${ }^{3}$ University of Groningen, University Medical Center Groningen, Tuberculosis Center Beatrixoord, Haren, The Netherlands. ${ }^{4}$ University of Groningen, University Medical Center Groningen, Dept of Pulmonary Diseases and Tuberculosis, Groningen, The Netherlands. ${ }^{5}$ National Tuberculosis Reference Laboratory, National Institute for Public Health and the Environment (RIVM), Bilthoven, The Netherlands. ${ }^{6}$ Radboud University Nijmegen Medical Center, Depts of Pulmonary Diseases and Medical Microbiology, Nijmegen, The Netherlands. ${ }^{7}$ University of Groningen, University Medical Center Groningen, Dept of Medical Microbiology and Infection Prevention, Groningen, The Netherlands.

${ }^{8}$ University of Groningen, University Medical Center Groningen, Dept of Internal Medicine, Groningen, The Netherlands.
\end{abstract}

Correspondence: Jan-Willem C. Alffenaar, University of Groningen, University Medical Center Groningen, Dept of Hospital and Clinical Pharmacy, PO box 30.001, 9700 RB Groningen, The Netherlands.

E-mail: j.w.c.alffenaar@umcg.nl

ABSTRACT Multidrug-resistant tuberculosis (MDR-TB) is a major global health problem. The loss of susceptibility to an increasing number of drugs behoves us to consider the evaluation of non-traditional anti-tuberculosis drugs.

Clarithromycin, a macrolide antibiotic, is defined as a group 5 anti-tuberculosis drug by the World Health Organization; however, its role or efficacy in the treatment of MDR-TB is unclear. A systematic review of the literature was conducted to summarise the evidence for the activity of macrolides against MDR-TB, by evaluating in vitro, in vivo and clinical studies. PubMed and Embase were searched for English language articles up to May 2014.

Even though high minimum inhibitory concentration values are usually found, suggesting low activity against Mycobacterium tuberculosis, the potential benefits of macrolides are their accumulation in the relevant compartments and cells in the lungs, their immunomodulatory effects and their synergistic activity with other anti-TB drugs.

A future perspective may be use of more potent macrolide analogues to enhance the activity of the treatment regimen.

$@$ ERSpublications

Macrolides deserve more research interest for use against MDR-TB as results appear to be more promising than thought http://ow.ly/LDFjp 


\section{Introduction}

Multidrug-resistant tuberculosis (MDR-TB) still remains a major problem in global health. MDR-TB is caused by Mycobacterium tuberculosis that is resistant to at least isoniazid and rifampicin [1-3], depriving the patient of the two most effective drugs used. MDR-TB should be treated by a regimen containing at least four anti-tuberculosis (TB) drugs to which the bacterium is susceptible [4]. By the end of 2012 it was estimated that globally $3.7 \%$ of treatment-naïve TB patients and $20 \%$ of previously treated cases have MDR-TB [5], in 2013 this number was adjusted to $3.5 \%$ and $20.5 \%$, respectively [6]. The problem of drug resistant $M$. tuberculosis is increasing, both in terms of loss of susceptibility to an increasing number of drugs, as well as in terms of the proportion and absolute numbers of TB patients with MDR-TB [7-9]. Evaluation of the effectiveness of non-traditional or newly synthesised anti-tubercular drugs is therefore necessary. Clarithromycin (CLR), a macrolide, is classified as a "group 5" anti-TB drug, defined by the World Health Organization (WHO) as anti-TB drugs with unclear efficacy or an unclear role in the treatment of MDR-TB [4]. Macrolides are a group of antibiotics already widely used for the treatment of upper and lower respiratory tract infections. The molecular structure consists of a macrocyclic lactone ring with various amino sugar side groups. The macrolides in clinical use have a 14-, 15- and 16-membered lactone ring. They can be subdivided into natural products (erythromycin) and semi-synthetic derivatives (CLR, roxithromycin and ketolides) $[10,11]$. Macrolides are considered bacteriostatic agents in clinically useful concentrations and act by inhibiting protein synthesis through binding to the large 50 S ribosomal subunit $[2,12]$.

Some semi-synthetic macrolides have already shown efficacy against, and are part of standard therapy against, many nontuberculous mycobacteria [13], such as Mycobacterium leprae [14-16], Mycobacterium ulcerans $[17,18]$ and Mycobacterium avium [19, 20]. As stated in the WHO guidelines, little is known about the effectiveness of macrolides against M. tuberculosis [4]. We, therefore, conducted a systematic review of the literature to summarise the evidence for the activity of macrolides against MDR-TB, by evaluating in vitro, in vivo and clinical studies.

\section{Mechanism of action}

Macrolides, azalides and ketolides exert antibacterial activity by inhibiting protein synthesis through binding to the $50 \mathrm{~S}$ ribosomal subunit near the peptidyl transferase centre in Deinococcus radiodurans [21, 22] and Mycobacterium smegmatis [23]. They block the exit tunnel through which newly synthesised peptides move away from the peptidyl transferase centre, obstructing elongation of the peptide chain. It is thought that a second inhibitory activity blocks the assembly of rRNAs and r-proteins resulting in inhibition of formation of the large $50 \mathrm{~S}$ ribosomal subunit [24, 25]. It is not clear whether these mechanisms of action are the same for slowly growing mycobacteria as for M. smegmatis. One study showed that CLR disorganises the outer wall layer and cytoplasmic membranes in the M. avium cell envelope eventually leading to bacterial death [26]. Furthermore, it is thought that macrolides tend to exert immunomodulatory effects in respiratory diseases, independent of their antimicrobial effects [27]. The putative immunomodulatory mechanisms of macrolides include improvement of the primary defence reaction, decreasing neutrophil migration and infiltration into respiratory epithelium, downregulation of the inflammatory cascade, reduction in generation of oxygen-free radicals, modulation of chemokine release and decreasing excessive pro-inflammatory mediators and cytokine production. These effects are hypothesised to control exacerbations of various chronic respiratory diseases, like cystic fibrosis, chronic obstructive pulmonary disease, asthma, chronic bronchitis and diffuse panbronchiolitis, and probably contribute to the therapeutic effect. Furthermore, it has been shown that macrolides reduce airway hyperresponsiveness and improve pulmonary function [28-34].

\section{Methods}

Articles concerning macrolide activity and/or efficacy against multidrug-resistant M. tuberculosis retrieved using a specific search strategy, described in the following section, were reviewed.

\section{Search strategy}

To retrieve relevant articles that describe the activity of macrolides against multidrug-resistant M. tuberculosis, the following search terms were used to search in PubMed: ("Tuberculosis"[Mesh] OR tubercul $^{*}[\mathrm{tw}]$ ) AND (“Tuberculosis, Multidrug-Resistant”[Mesh] OR MDR*[tw] OR resist*[tw]) AND ("Macrolides"[Mesh] OR "telithromycin" [Supplementary Concept] OR “cethromycin" [Supplementary Concept] OR "CEM 101" [Supplementary Concept] OR erythromycin*[tw] OR telithromycin* $\left.{ }^{*} \mathrm{tw}\right]$ OR cethromycin ${ }^{\star}[\mathrm{tw}]$ OR Clarithromycin ${ }^{\star}[\mathrm{tw}]$ OR Azithromycin ${ }^{\star}[\mathrm{tw}]$ OR Roxithromycin ${ }^{\star}[\mathrm{tw}] \mathrm{OR}$ telithromycin ${ }^{*}[\mathrm{tw}]$ OR solithromycin*[tw] OR cethromycin*[tw]). In Embase, the same search term was inserted as follows: "tuberculosis"/exp OR tubercul*:ab,ti OR "multidrug resistant tuberculosis"/exp AND (mdr*:ab,ti OR resist*:de,ab,ti) AND ("macrolide"/exp OR "ketolide"/exp OR erythromycin*:ab,ti OR clarithromycin*:ab,ti OR azithromycin*:ab,ti OR roxithromycin*ab,ti OR telithromycin*:ab,ti OR 
solithromycin*:ab,ti OR cethromycin*:ab,ti). Relevant articles were included up until May 2014 and supplemented by hand-searching the reference lists of identified eligible studies. Articles described as editorials, comments, reviews or case studies were not included. Citations were screened independently by more than one reviewer, examining titles and abstracts to identify potentially relevant articles. Differences were resolved by consensus. Extracted data from the searches were entered into Excel (Microsoft, Redmond, WA, USA), duplicates were removed and original English language articles were then retrieved and the full text screened for final inclusion and data extraction. No attempts were made to obtain missing data from the researchers of eligible studies.

\section{Selection criteria}

Studies were eligible for inclusion if the activity of a specific macrolide against multidrug resistant M. tuberculosis was evaluated in in vitro, in vivo or clinical studies. In vitro studies were included if the method of testing susceptibility of MDR-TB to macrolides was adequately described, including the radiometric BACTEC method (Becton Dickinson Microbiologic Systems, Detroit, MI, USA), microplate Alamar blue assay (MABA) (Accumed International, Westlake, OH, USA), proportion methods, and broth dilution and microdilution methods in different media. In vitro studies were subsequently subdivided into studies that evaluated extracellular and intracellular susceptibility, and drug-drug synergy. As data on in vitro intracellular susceptibility testing of MDR-TB strains was lacking, data from experiments with drug-susceptible strains were included.

The susceptibility of MDR-TB to anti-TB drugs is described by the minimum inhibitory concentration (MIC). The MIC is the lowest concentration that prevents growth of bacteria (visually or photometrically determined). MIC90 is the MIC that inhibits $90 \%$ of the isolates or strains. A MIC range of $2-4 \mu \mathrm{g} \cdot \mathrm{mL}^{-1}$ is defined as being a low MIC value, $8-16 \mu \mathrm{g} \cdot \mathrm{mL}^{-1}$ as intermediate and $\geqslant 16 \mu \mathrm{g} \cdot \mathrm{mL}^{-1}$ as a high MIC value. In addition, studies are included that present data on susceptibility as viable count enumeration (VCE) (i.e. bactericidal activity of a drug) or number of colony-forming units (CFU) [35].

To assess whether macrolides show synergy, indifference or antagonism with other anti-TB drugs, studies describing the combination of a macrolide and any drug used for the treatment of MDR-TB as defined by the WHO treatment guidelines were included. Often the fractional inhibitory concentration (FIC) is used to describe the interaction between two antimicrobial agents. The FIC is determined as follows:

$$
\mathrm{FICA}=(\mathrm{MICA}, \text { combination/MICA, alone })+(\mathrm{MICB}, \text { combination/MICB, alone })
$$

where FICA is the FIC of drug A, MICA, combination is the MIC of drug A when combined with a second drug, MICA, alone is the MIC of drug A when used alone, MICB, combination is the MIC of drug B when combined with a second drug, MICB, alone is the MIC of drug B when used alone. The FIC was interpreted as follows: FIC $\leqslant 0.5$ suggests synergistic activity, FIC $1-4$ suggests indifference and FIC $>4$ suggests antagonism [36, 37].

In vivo animal studies were included if adequate data were provided on measures of effect in comparison with control (mean $\log _{10}$ CFUs in spleen and lungs, mortality, and weight of spleen), type of TB strain, type of animal strain, type of infection model, drug doses, treatment duration and organs studied. In addition, if synergism between two drugs was described, information or data on the difference between the mean $\log _{10}$ CFUs of the combination of drugs and the lowest mean $\log _{10}$ CFU of one drug used alone had to be reported.

Clinical studies reporting on treatment of MDR-TB using macrolides were included if they at least reported data on the treatment regimen (including CLR) and treatment outcome (efficacy and/or adverse events) or pharmacokinetics.

\section{Results}

In total 1713 articles were retrieved from the initial search (fig. 1). After screening of titles and abstracts, only 169 articles were considered eligible for evaluation. Ultimately, 37 articles were included describing in vitro susceptibility testing of macrolides against MDR-TB strains, in vitro synergy testing with any drugs for the treatment of MDR-TB as defined by the WHO treatment guidelines, in vivo studies and clinical studies.

\section{In vitro studies}

\section{Extracellular susceptibility testing}

Table 1 presents the results for studies concerning extracellular susceptibility testing. Most studies performed drug susceptibility testing against $M$. tuberculosis by determining the MIC using the radiometric BACTEC 460 method [36-41, 44-46]. Often a concentration range up to $16 \mu \mathrm{g} \cdot \mathrm{mL}^{-1}$ was used $[36,37,39$, 41]. Overall, studies have shown no susceptibility of MDR-TB strains to CLR, with all studies reporting 


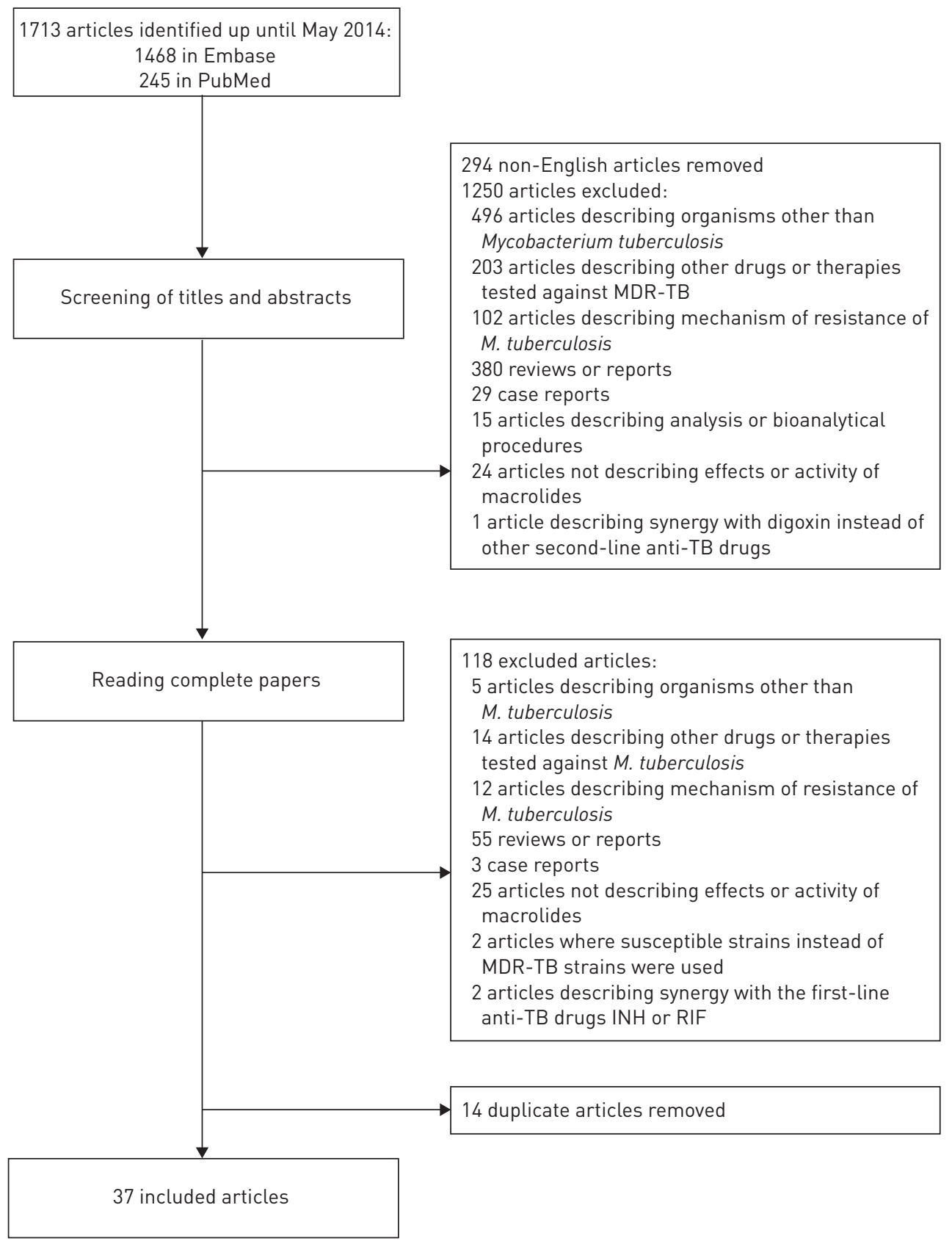

FIGURE 1 Flow chart of the selection procedure. MDR-TB: multidrug-resistant tuberculosis; TB: tuberculosis; INH: isoniazid; RIF: rifampicin.

high MIC values [36-41]. This was also observed for roxithromycin, azithromycin (AZI) [41] and the metabolite of CLR 14-hydroxyclarithromycin [36]. However, in another study the MIC values for CLR against $69 \mathrm{MDR}-\mathrm{TB}$ isolates were $<4 \mu \mathrm{g} \cdot \mathrm{mL}^{-1}$, with only one strain being resistant [46].

In addition, other studies have reported rather high MICs obtained using the proportion method with Middlebrook 7H10 medium. MICs in these studies also indicate that CLR [43] and roxithromycin [45] are unsuitable alternative agents when used alone against MDR-TB, based on high MIC90 and MIC values, respectively. CLR, erythromycin and AZI were also shown to be inactive against 17 MDR-TB isolates when using the broth dilution method in 7H9 Middlebrook medium [42]. The broth dilution and microdilution method were also used in 7HSF medium, which resulted in MICs for CLR of $20 \mu \mathrm{g} \cdot \mathrm{mL}^{-1}$ [48] and $25 \mu \mathrm{g} \cdot \mathrm{mL}^{-1}$ [49]. Furthermore, the use of the MABA with three MDR-TB strains resulted in MIC values in the range of $2-32 \mu \mathrm{g} \cdot \mathrm{mL}^{-1}$. When tested against the virulent $\mathrm{H} 37 \mathrm{Rv}$ strain $\mathrm{ROX}, \mathrm{AZI}$ and telithromycin 


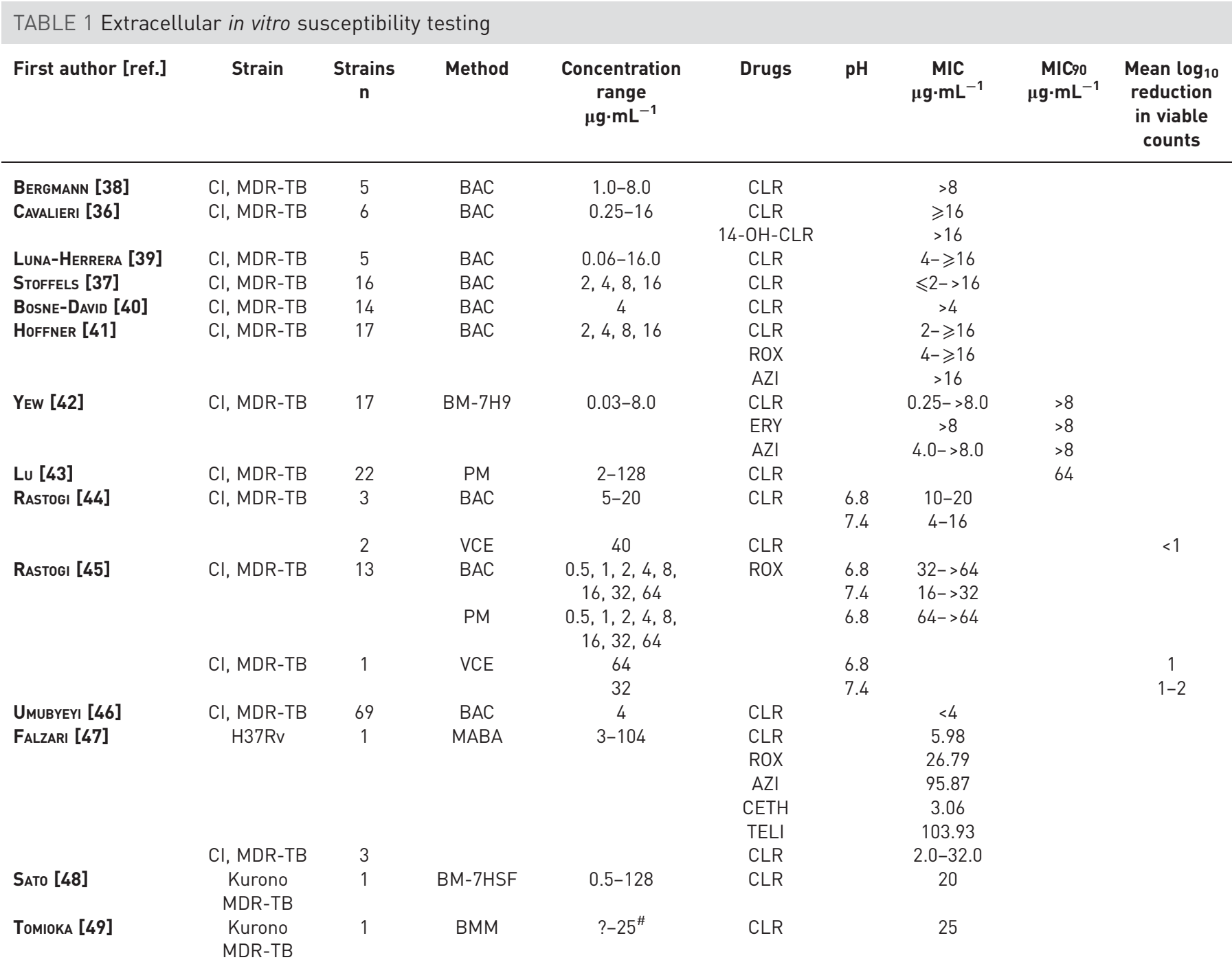

MIC: minimal inhibitory concentration; MIC90: MIC that inhibits $90 \%$ of the isolates or strains; $\mathrm{Cl}$ : clinical isolate; MDR-TB: multidrug-resistant tuberculosis; BAC: Radiometric BACTEC TB 460; CLR: clarithromycin; 14-OH-CLR: 14-hydroxyclarithromycin; ROX: roxithromycin; AZI: azithromycin; BM-7H9: broth dilution method with 7H9 medium; ERY: erythromycin; PM: proportion method with Middlebrook 7H10 agar; VCE: viable count enumeration; MABA: microplate Alamar blue assay; CETH: cethromycin; TELI: telithromycin; BM-7HSF: broth dilution method with 7HSF medium; BMM: broth microdilution method using 7SHF medium. " : tested range described in the methods section.

resulted in high MIC values of 6-96 $\mu \mathrm{g} \cdot \mathrm{mL}^{-1}$, while CLR gave a MIC of $\sim 6 \mu \mathrm{g} \cdot \mathrm{mL}^{-1}$ and cethromycin yielded a lower MIC of $3 \mu \mathrm{g} \cdot \mathrm{mL}^{-1}$ [47].

Some studies using the BACTEC method or proportion method did not report their results in terms of MICs, but as the number of strains that were resistant or susceptible to a macrolide. Different studies have reported $11(84.6 \%)$ out of 13 [50], $19(63.3 \%)$ out of 30 [51], six (24\%) out of 25 [52] or eight (10.3\%) out of 78 [53] MDR-TB strains as being resistant to CLR using the radiometric BACTEC method. Another study reported that five (6.9\%) out of $72 \mathrm{MDR}-\mathrm{TB}$ strains were resistant to CLR/AZI as estimated using the BACTEC or proportion method [54]. However, using the proportion method 52 (51.5\%) out of 101 [55] and 517 (88.1\%) out of 587 [56] clinical isolates from MDR-TB patients were susceptible to CLR. Furthermore, in this study four (50\%) out of eight clinical isolates from extensively drug-resistant TB (XDR-TB) patients were susceptible to CLR [56].

Some studies have addressed the influence of $\mathrm{pH}$ on in vitro susceptibility testing using the radiometric BACTEC method. MICs of CLR and roxithromycin appeared to be lower at $\mathrm{pH} 7.4$ compared with the routine $\mathrm{pH}$ of $6.8[44,45]$. This was also seen when the bacterial viability was determined by VCE; roxithromycin appeared to be more active at $\mathrm{pH} 7.4$ compared with $\mathrm{pH} 6.8$ [45]. 
Irrespective of the methods used, all studies show high MIC values, suggesting low susceptibility of MDR-TB to macrolides.

\section{Intracellular susceptibility testing}

Results for intracellular susceptibility testing are presented in table 2. Most studies assessed the intracellular susceptibility of bacteria to macrolides by counting CFU or MIC determination in M. tuberculosis-infected macrophages, alveolar cells and alveolar macrophages [39, 44, 47-49]. A dose-dependent inhibition of growth was observed for CLR against MDR-TB, H37Rv and two clinical isolates in J774A.1 mouse macrophages, with a 2-3 log reduced growth [39]. CLR and cethromycin showed a more modest bacteriostatic effect against drug-susceptible strains in J774A.1 mouse macrophages [47] and human macrophages [44], with a reduction of $<1 \log _{10}$ CFU. Furthermore, CLR displayed a modest inhibition of growth of the Kurono MDR-TB strain in Mono-Mac-6 human monocytic cell lines (MM6) and A549 type-II human alveolar epithelial cell lines (A549-II) [48, 49]. In the same study, MICs of 5 and $2.5 \mu \mathrm{g} \cdot \mathrm{mL}^{-1}$ were determined in MM6 and A549-II, respectively [48].

Thus, overall CLR resulted in the reduction of intracellular CFU counts.

\section{Synergy testing}

Results for synergy testing are presented in table 3. The combination of CLR and rifabutin resulted in a FIC of 1-2, suggesting indifference [38]. When CLR was combined with ethambutol (EMB) a synergistic effect was observed $[36,40]$. CLR and AZI also acted synergistically with spectinomycin against the drug susceptible H37Rv strain, FICs were all $\leqslant 0.5$. When tested within macrophages CLR showed synergy with spectinomycin, but AZI did not [58]. Finally, synergy was also observed between CLR and linezolid (LZD) with FICs in the range of $0.32-0.37$ and $0.375-1.0$ using the mycobacteria growth indicator tube 960 system (MGIT) (Becton Dickinson) and the absolute concentration method, respectively [57].

Thus CLR shows promising synergistic results when combined with other second line anti-TB drugs.

In vivo studies

Results from in vivo studies are presented in table 4. In murine infection models CLR showed modest activity, resulting in inhibition of growth of M. tuberculosis and MDR-TB. CLR reduced CFU counts in spleens and lungs compared with those of mice given no CLR treatment [47, 59]. CLR showed less activity against organisms in the lungs than organisms in the spleens. In another study, CLR showed complete protection from death since none of the CLR treated mice in the first experiment died during 8 weeks of treatment, whereas all non-treated control mice died within the same period. Furthermore, in the second arm of this study, in which controls did survive up to 4 weeks, CLR reduced mean CFU counts in both lungs and spleens compared with controls [39]. Also, CLR was given in combination with streptomycin or

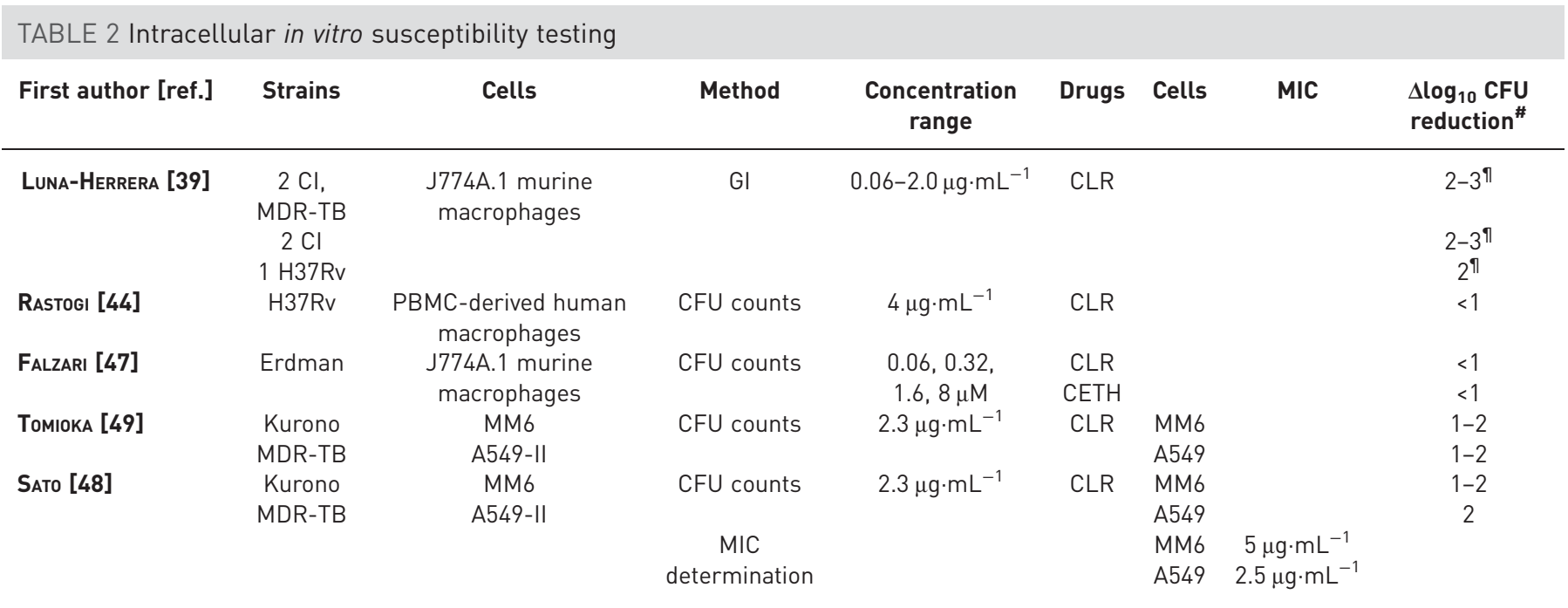

MIC: minimal inhibitory concentration; $\mathrm{Cl}$ : clinical isolate; MDR-TB: multidrug-resistant tuberculosis; J774A.1: murine monocyte macrophage cell line; GI: growth index; CLR: clarithromycin; PBMC: peripheral blood monocytic cells; CETH: cethromycin; MM6: Mono-Mac-6 human monocytic cell line; A549-II: type II human alveolar epithelial cell lines. \#: results are read from graphs, therefore a certain error must be taken into account; ${ }^{\text {ๆ: }}$ not a $\log _{10}$ CFU reduction but $\log _{10}$ reduced growth measured using growth index. 
TABLE 3 In vitro synergy of clarithromycin (CLR) and other second-line tuberculosis drugs

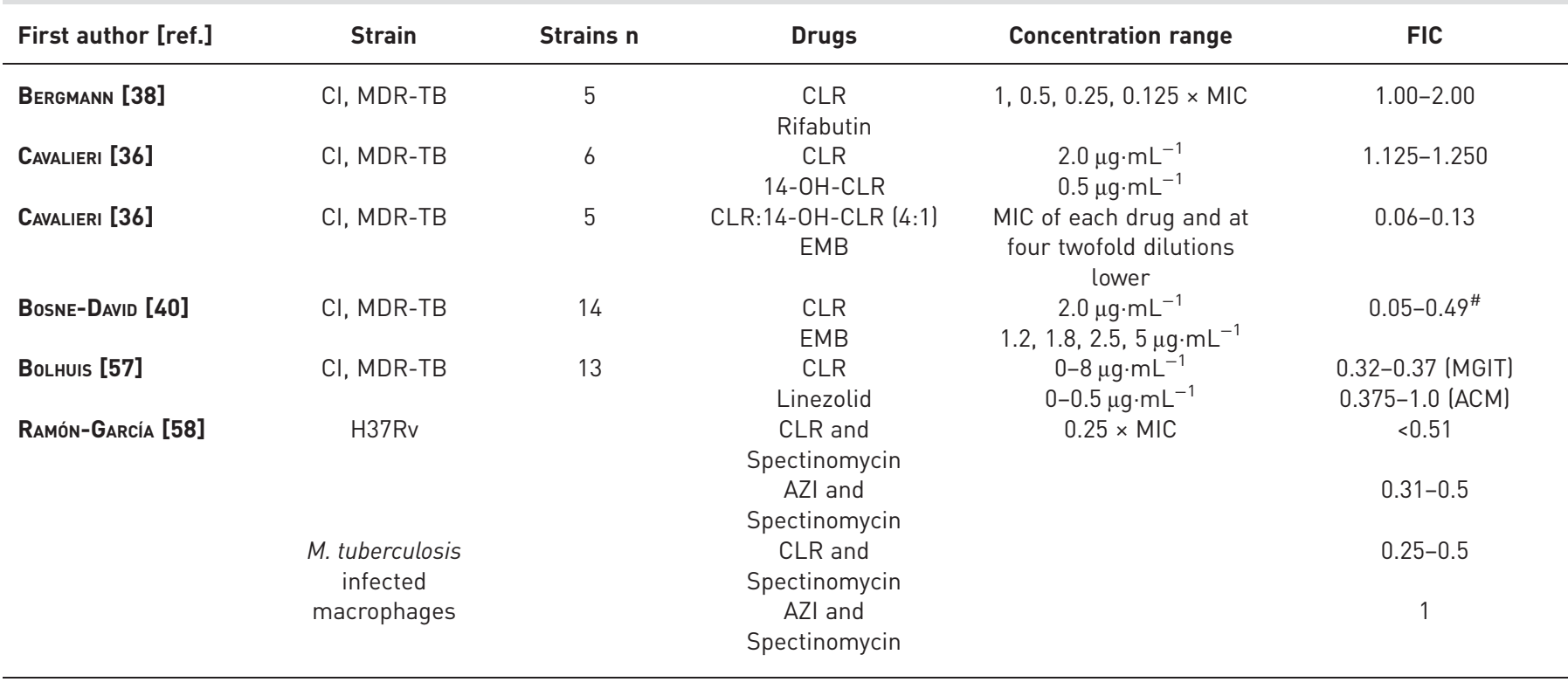

FIC: fractional inhibitory concentration; $\mathrm{Cl}$ : clinical isolate; MDR-TB: multidrug-resistant tuberculosis; MIC: minimal inhibitory concentration; 14-OH-CLR: 14-hydroxyclarithromycin; EMB: ethambutol; MGIT: Mycobacteria Growth indicator tube (MGIT) 960 system; ACM: absolute concentration method; AZI: azithromycin; M. tuberculosis: Mycobacterium tuberculosis. " : not measured by FIC but by X/Y quotient.

thioacetazone. No significant difference in CFU was observed in mice treated with streptomycin alone or with a combination of streptomycin and CLR. However, activity of the combination of thioacetazone plus CLR was slightly better than when the drugs were given separately, with CLR having slightly better activity than thioacetazone. In another study, mortality rate decreased by $60.8 \%$ and a $125 \mathrm{mg}$ difference in spleen weight was observed when CLR treated mice were compared with controls [60]. CFU counts in this study were difficult to compare, since the high mortality rate in the control group led to grossly underestimated CFU counts.

Overall CLR reduces mortality and provides protection from death when used in TB-infected mice, reducing mean CFU counts.

\section{Clinical studies}

Data on the clinical use of macrolides for the treatment of MDR-TB is scarce. Most studies evaluating MDR-TB treatment do not include patients with CLR or do not present the individual data. One study described the influence of CLR on LZD exposure in five MDR-TB patients. While $250 \mathrm{mg}$ CLR once daily had no statistically significant effect, $500 \mathrm{mg}$ of CLR significantly increased LZD serum exposure by a median of $44 \%$ compared with baseline [61]. Another retrospective study reported that when CLR was included in a salvage regimen, this did not necessarily result in improved culture conversion [62]. However, it is difficult to estimate the impact of the data from this study since only 14 patients with a complex medical history received CLR in their salvage regimen.

A number of studies also reported inclusion of macrolides in a treatment regimen for MDR- or XDR-TB, where some studies also included observed adverse events. The frequency with which macrolides were prescribed varied, depending on the type of resistance (MDR-TB or XDR-TB). One meta-analysis shows that macrolides given in the clinic do not result in superior treatment outcomes compared with other group 5 drugs [63]. Three (0.6\%) out of 481 MDR-TB patients received CLR over a period from 1992-2002 in South Africa [64]. 121 (11.6\%) out of 1047 MDR-TB patients from Latvia, Lima, Manila and Tomsk received CLR in their treatment regimen [65]. 72 (34.1\%) out of 211 MDR-TB patients received CLR and $15(7.1 \%)$ out of 211 received roxithromycin as part of their treatment [66]. In four out of 13 patients CLR was used over a period of 3.6 years [67]. Among 655 subjects evaluated, CLR was prescribed 44 times and roxithromycin once, furthermore CLR was discontinued twice (4.5\%) due to adverse drug reactions of the gastrointestinal tract and headache [68]. 28 (10.6\%) out of 263 MDR-TB patients received CLR in an anti-TB regimen, where CLR was withdrawn in two patients due to severe side-effects [69]. One (5.9\%) patient discontinued treatment with CLR due to nephrotoxicity out of 


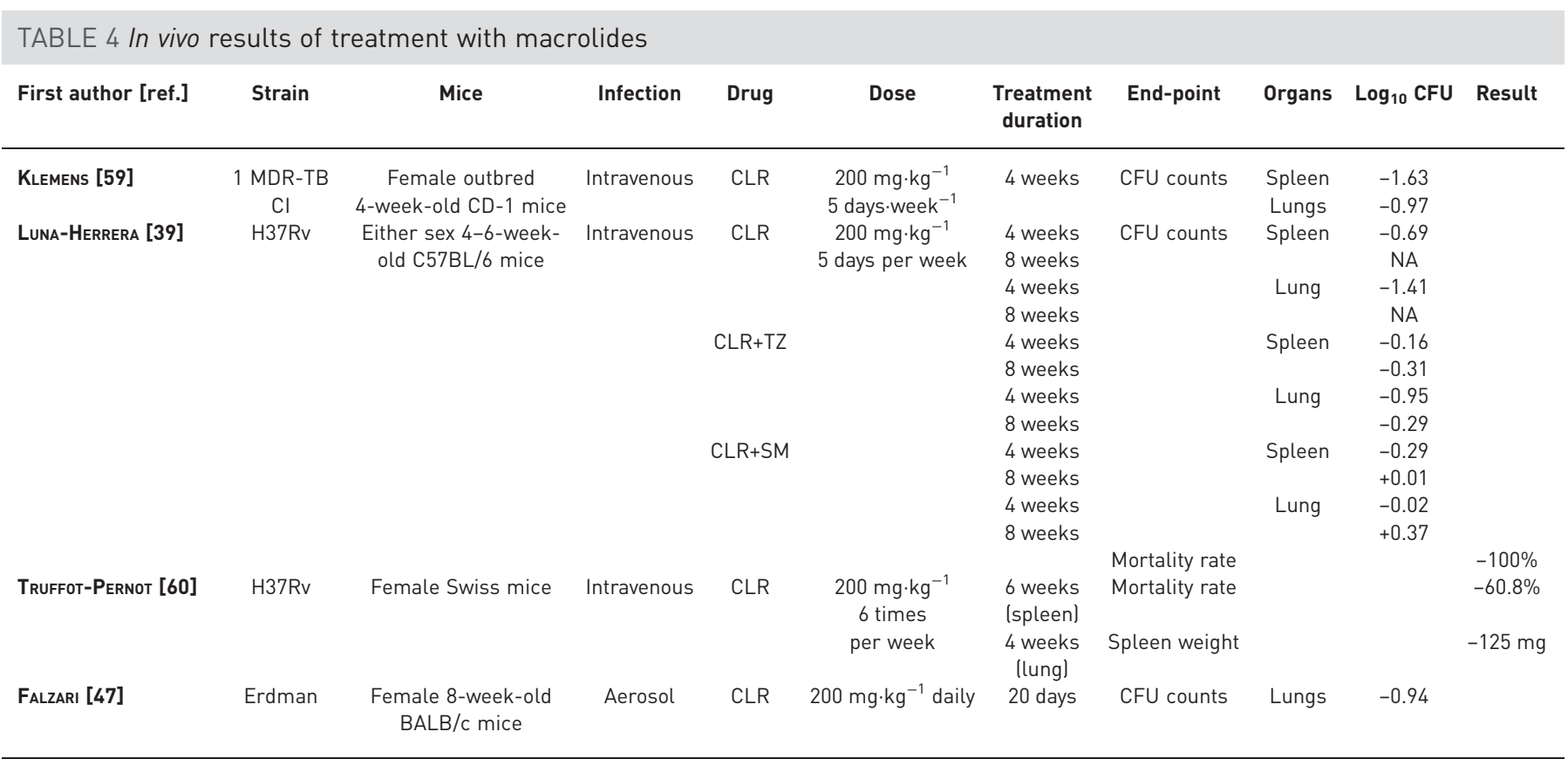

17 patients given CLR treatment [51]. One (4\%) out of 25 received treatment with CLR over a period of 2 years in Madrid [52]. Furthermore, another article assessed the treatment outcome in relation to inclusion of CLR in the previous TB regimen. Six (21.4\%) out of 28 patients with a favourable outcome had CLR in their previous TB regimen, as opposed to $21(18.4 \%)$ out of 114 patients with a poor outcome having CLR in their previous TB regimen. Cure and treatment completion was defined as a favourable outcome and treatment failure, death and default were defined as a poor outcome. Finally, in a study among UK children with MDR-TB, in three out of the 17 children evaluated CLR was included in the regimen [70].

Macrolide use appears more frequent in patients with XDR-TB. 21 (44.6\%) out of 47 XDR-TB patients received CLR, with a treatment duration of 14.5 (11.6-18.9) months [71]. Another study reports that 23 (53.5\%) out of 43 XDR-TB patients received CLR and six (14.0\%) out of 43 received roxithromycin in their treatment regimen [66]. Furthermore, 11 (9.6\%) out of 115 XDR-TB patients received AZI and 77 (67\%) out of 115 XDR-TB patients received CLR, no severe adverse events were observed for both drugs [72].

These studies demonstrate that macrolides are included treatment regimens when strains become more drug-resistant (MDR-TB and XDR-TB), when other drug treatment options fail.

\section{Discussion}

Macrolides have high MIC values in M. tuberculosis isolates when evaluated in vitro [36-45, 47-49]. However, some studies reported promising results that might contribute to clinical activity in the sense of macrolides exerting bacteriostatic effects in vitro $[39,45,48,49]$ and in vivo $[39,47,59]$. Furthermore, a decrease in mortality in murine infection models $[39,60]$ and synergistic effects when CLR was combined in vitro with second-line anti-TB drugs $[36,37,40,57,73]$ were seen. Also, an interaction was reported in a clinical study [61], suggesting an increase of LZD serum concentration when combined with CLR.

Despite high MIC values, some bronchopulmonary pharmacokinetic studies demonstrated that high concentrations of CLR were achieved in alveolar macrophages and epithelial lining fluid (ELF), with high ratios of concentrations in ELF and alveolar macrophages compared with that in plasma assessed in healthy volunteers [74-76]. These results suggest that high concentrations of CLR are achieved in ELF and alveolar macrophage cells, which exceed the in vitro MIC values by several fold. Especially in alveolar macrophages, the concentrations of CLR reached may exceed MIC values that could help eradication of MDR-TB. Therefore, the high MIC values obtained from extracellular in vitro studies may not necessarily indicate the full potential efficacy of CLR against MDR-TB, since this drug is not equally distributed in the body but accumulates in relevant target compartments and target cells in the lungs. This should be considered when giving the drug to a patient with extrapulmonary TB. In intracellular susceptibility 
studies CLR resulted in reduction of CFU counts [39, 48, 49]. This effect can be explained by other studies describing the ability of CLR to accumulate in phagocytic cells, resulting in high intracellular to extracellular concentration ratios $[77,78]$.

An important aspect seen for CLR is the synergistic effect when it is combined with other anti-TB drugs. This synergistic effect may be substance-dependent with a preference towards drugs with a smaller molecular volume, like EMB. If CLR affects the outer cell wall layer and cytoplasmic membrane of M. tuberculosis, as it does in M. avium [26], it is possible that the permeability for other drugs increases, resulting in a synergistic effect. Unfortunately no data is available on combination with kanamycin, amikacin, moxifloxacin and levofloxacin. For this reason future prospects for CLR could also include using CLR in combination regimens together with more vigorous bactericidal drugs to clear MDR-TB. In this setting CLR may serve as a companion drug, increasing permeability to other antimicrobial agents and exerting its immunomodulatory effects. However, it should not be counted as or included among the drugs making up the standard regimen [4]. Furthermore, besides the observed in vitro synergistic effect of CLR and LZD [57], one clinical study also described an interaction between CLR and LZD suggesting an increase of LZD serum concentration when combined with CLR [61]. This could potentially be cost saving, because a reduced dose of the very expensive drug LZD can be used to reach similar drug exposure and this should be verified on a case by case basis by measuring LZD levels. The observed interaction also has a downside since the occurrence of major adverse events associated with LZD, such as anaemia and peripheral neuropathy, are higher when serum concentrations of LZD are higher. Therefore, this necessitates caution when prescribing CLR simultaneously with LZD [61].

Despite high MIC values obtained by MIC testing, bacteriostatic effects were seen with VCE and CFU counts in vitro $[39,45,48,49]$ and in vivo $[39,47,59]$. Furthermore, in vivo results showed a decrease in mortality when macrolides were added to the treatment regimen $[39,60]$. These findings suggest CLR may serve as a lead compound for exploring the possibilities of structural modifications to create more potent derivatives. This strategy is already used for several other TB drugs. SQ109, the synthetic analogue of EMB, shows promising in vitro and in vivo anti-TB activity [79] and has completed three phase 1 studies. LZD, belonging to the oxazolidinone class of antimicrobials, has shown efficacy against MDR-TB [80, 81], but its associated serious adverse events have prompted development of new oxazolidinones, such as PNU-100480 [82] and AZD5847 [83]. Also in the case of metronidazole, a member of the nitroimidazole class of antimicrobials, the structurally related nitroimidazopyrans, PA824 and OP-67683, were found to possess potent activity against MDR-TB $[84,85]$.

In the large group of macrolides a new generation, known as the ketolides, has emerged. The ketolides include telithromycin, cethromycin and solithromycin. There are data suggesting that the mechanism of action of these derivatives is slightly different from earlier generations of macrolides. Ketolides appear to allow more proteins to pass through the ribosome during protein synthesis. By contrast, the macrolides lead to a complete or near-complete inhibition of protein synthesis. This partial inhibition of protein synthesis by ketolides is likely to cause more cellular deregulation, which appears to have a stronger bactericidal effect [86]. From these ketolides, fluoroketolides like K-1602, K-1636, K-1835 and K-1804 have been synthesised, which show improved MIC values against Escherichia coli, Staphylococcus aureus and Streptococcus pyogenes compared with erythromycin and the earlier generation ketolide, telithromycin [87]. This example shows the potential to improve antibacterial activity of analogues derived from a lead compound, which is initially classified as bacteriostatic. In addition, other fluoroketolides, like HMR-3562 and HMR-3787, have shown activity against several respiratory tract pathogens, like S. aureus, pneumococci and Haemophilus species [88]. However, for the ketolides discussed in this review it should be noted that the US Food and Drug Administration (FDA) strengthened their warning on the risk of liver toxicity when using telithromycin, the first FDA-approved ketolide [89]. The drugs should be used cautiously since de novo hepatotoxicity developed within a few days following treatment with telithromycin in three individuals with previously normal liver test results [90]. The authors judged the adverse drug reactions as probably but not definitely related to telithromycin. Moreover, the FDA strengthened warning regarding telithromycin-related adverse events including visual disturbances and loss of consciousness [89, 91].

Further limitations of macrolide treatment are on the grounds of adverse drug reactions and drug-drug interactions. In case reports macrolides are found to be associated with prolongation of the QT interval and Torsades de Pointes, prolonging the repolarisation period of the action potential by blocking the HERG potassium channels, similar to class III antiarrhythmics [92]. The European Society of Cardiology considers erythromycin and CLR as drugs that have the greatest potential for generating Torsades de Pointes and QT interval prolongation [93]. The proarrhythmic potential of the individual macrolide antibiotics is different as reported in a retrospective analysis of case reports that appeared in the FDA adverse event reporting system on macrolides and Torsade de Pointes. In 156 patients, proarrythmic potential was associated with erythromycin in 53\% of cases and with CLR in 36\% of cases, and $11 \%$ 
occurred in azalide-treated patients [93]. Since other MDR-TB drugs like moxifloxacin [94], delamanid [95] and bedaquiline [96] are also known to lengthen QT interval, adding macrolides to a MDR-TB regimen could increase the risk of cardiovascular events and mortality.

To summarise, although high MIC values are usually found for M. tuberculosis the potential benefits of macrolides are: their accumulation in relevant compartments and cells in the lungs; their immunomodulatory effects; and their ability to disorganise the cell envelope, leading to increased permeability to other bactericidal drugs. To conclude, macrolides deserve more research interest than they currently attract, as the current results, demonstrating synergistic effects, decreased mortality in vivo and inclusion in the treatment regimens of patients with MDR-TB, appear to be more promising than previously thought. The future for potential novel drugs for MDR-TB is brighter than ever with more drugs in the pipeline than over the past decades. Among these may be a more active macrocyclic drug with beneficial secondary effects to enhance the activity of the treatment regimen.

\section{References}

1 World Health Organization. Towards universal access to diagnosis and treatment of multidrug-resistant and extensively drug-resistant tuberculosis by 2015. WHO progress report 2011. Geneva, World Health Organization, 2011.

2 Caminero JA, Sotgiu G, Zumla A, et al. Best drug treatment for multidrug-resistant and extensively drug-resistant tuberculosis. Lancet Infect Dis 2010; 10: 621-629.

3 Dooley KE, Obuku EA, Durakovic N, et al. World Health Organization group 5 drugs for the treatment of drug-resistant tuberculosis: unclear efficacy or untapped potential? J Infect Dis 2013; 207: 1352-1358.

4 World Health Organization. Guidelines for the programmatic management of drug-resistant tuberculosis. 2011 update. Geneva, World Health Organization, 2011.

5 World Health Organization. Global tuberculosis report 2012. Geneva, World Health Organization, 2012.

6 World Health Organization. Global tuberculosis report 2014. Geneva, World Health Organization, 2014.

7 Zumla A, Abubakar I, Raviglione M, et al. Drug-resistant tuberculosis - current dilemmas, unanswered questions, challenges, and priority needs. J Infect Dis 2012; 205: Suppl. 2, S228-S240.

8 Cohen T, Jenkins HE, Lu C, et al. On the spread and control of MDR-TB epidemics: an examination of trends in anti-tuberculosis drug resistance surveillance data. Drug Resist Updat 2014; 17: 105-123.

9 Perdigão J, Macedo R, Silva C, et al. From multidrug-resistant to extensively drug-resistant tuberculosis in Lisbon, Portugal: the stepwise mode of resistance acquisition. J Antimicrob Chemother 2013; 68: 27-33.

10 Jalava J, Marttila H. Application of molecular genetic methods in macrolide, lincosamide and streptogramin resistance diagnostics and in detection of drug-resistant Mycobacterium tuberculosis. APMIS 2004; 112: 838-855.

11 Retsema J, Fu W. Macrolides: structures and microbial targets. Int J Antimicrob Agents 2001; 18: Suppl. 1, S3-10.

12 Buriánková K, Doucet-Populaire F, Dorson O, et al. Molecular basis of intrinsic macrolide resistance in the Mycobacterium tuberculosis complex. Antimicrob Agents Chemother 2004; 48: 143-150.

13 Griffith DE, Aksamit T, Brown-Elliott BA, et al. An official ATS/IDSA statement: diagnosis, treatment, and prevention of nontuberculous mycobacterial diseases. Am J Respir Crit Care Med 2007; 175: 367-416.

14 Ji B, Jamet P, Perani EG, et al. Bactericidal activity of single dose of clarithromycin plus minocycline, with or without ofloxacin, against Mycobacterium leprae in patients. Antimicrob Agents Chemother 1996; 40: 2137-2141.

15 Chan GP, Garcia-Ignacio BY, Chavez VE, et al. Clinical trial of clarithromycin for lepromatous leprosy. Antimicrob Agents Chemother 1994; 38: 515-517.

16 Grosset JH. Newer drugs in leprosy. Int J Lepr Other Mycobact Dis 2001; 69: S14-S18.

17 Nienhuis WA, Stienstra Y, Thompson WA, et al. Antimicrobial treatment for early, limited Mycobacterium ulcerans infection: a randomised controlled trial. Lancet 2010; 375: 664-672.

18 Alffenaar JW, Nienhuis WA, de Velde F, et al. Pharmacokinetics of rifampin and clarithromycin in patients treated for Mycobacterium ulcerans infection. Antimicrob Agents Chemother 2010; 54: 3878-3883.

19 Griffith DE, Wallace RJ Jr. New developments in the treatment of nontuberculous mycobacterial (NTM) disease. Semin Respir Infect 1996; 11: 301-310.

20 Fernandes $\mathrm{PB}$, Hardy $\mathrm{DJ}$, McDaniel $\mathrm{D}$, et al. In vitro and in vivo activities of clarithromycin against Mycobacterium avium. Antimicrob Agents Chemother 1989; 33: 1531-1534.

21 Schlünzen F, Harms JM, Franceschi F, et al. Structural basis for the antibiotic activity of ketolides and azalides. Structure 2003; 11: 329-338.

22 Schlünzen F, Zarivach R, Harms J, et al. Structural basis for the interaction of antibiotics with the peptidyl transferase centre in eubacteria. Nature 2001; 413: 814-821.

23 Pfister P, Jenni S, Poehlsgaard J, et al. The structural basis of macrolide-ribosome binding assessed using mutagenesis of 23S rRNA positions 2058 and 2059. J Mol Biol 2004; 342: 1569-1581.

24 Champney WS, Burdine R. Macrolide antibiotics inhibit 50S ribosomal subunit assembly in Bacillus subtilis and Staphylococcus aureus. Antimicrob Agents Chemother 1995; 39: 2141-2144.

25 Chittum HS, Champney WS. Erythromycin inhibits the assembly of the large ribosomal subunit in growing Escherichia coli cells. Curr Microbiol 1995; 30: 273-279.

26 Rastogi N, Labrousse V. Extracellular and intracellular activities of clarithromycin used alone and in association with ethambutol and rifampin against Mycobacterium avium complex. Antimicrob Agents Chemother 1991; 35: $462-470$.

27 Altenburg J, de Graaff CS, van der Werf TS, et al. Immunomodulatory effects of macrolide antibiotics - part 2: advantages and disadvantages of long-term, low-dose macrolide therapy. Respiration 2011; 81: 75-87.

28 Zarogoulidis P, Papanas N, Kioumis I, et al. Macrolides: from in vitro anti-inflammatory and immunomodulatory properties to clinical practice in respiratory diseases. Eur J Clin Pharmacol 2012; 68: 479-503.

29 Parnham MJ. Immunomodulatory effects of antimicrobials in the therapy of respiratory tract infections. Curr Opin Infect Dis 2005; 18: 125-131. 
Sharma S, Jaffe A, Dixon G. Immunomodulatory effects of macrolide antibiotics in respiratory disease: therapeutic implications for asthma and cystic fibrosis. Paediatr Drugs 2007; 9: 107-118.

31 Healy DP. Macrolide immunomodulation of chronic respiratory diseases. Curr Infect Dis Rep 2007; 9: 7-13.

32 Friedlander AL, Albert RK. Chronic macrolide therapy in inflammatory airways diseases. Chest 2010; 138: 1202-1212.

33 Ianaro A, Ialenti A, Maffia P, et al. Anti-inflammatory activity of macrolide antibiotics. J Pharmacol Exp Ther 2000; 292: 156-163.

34 Altenburg J, de Graaff CS, Stienstra Y, et al. Effect of azithromycin maintenance treatment on infectious exacerbations among patients with non-cystic fibrosis bronchiectasis: the BAT randomized controlled trial. JAMA 2013; 309: 1251-1259.

35 European Committee for Antimicrobial Susceptibility Testing (EUCAST) of the European Society of Clinical Microbiology and Infectious Dieases (ESCMID). EUCAST Definitive Document E.Def 1.2, May 2000: terminology relating to methods for the determination of susceptibility of bacteria to antimicrobial agents. Clin Microbiol Infect 2000; 6: 503-508.

36 Cavalieri SJ, Biehle JR, Sanders WE Jr. Synergistic activities of clarithromycin and antituberculous drugs against multidrug-resistant Mycobacterium tuberculosis. Antimicrob Agents Chemother 1995; 39: 1542-1545.

37 Stoffels K, Traore H, Vanderbist F, et al. The effect of combined tobramycin-clarithromycin on Mycobacterium tuberculosis isolates. Int J Tuberc Lung Dis 2009; 13: 1041-1044.

38 Bergmann JS, Woods GL. In vitro activity of antimicrobial combinations against clinical isolates of susceptible and resistant Mycobacterium tuberculosis. Int J Tuberc Lung Dis 1998; 2: 621-626.

39 Luna-Herrera J, Reddy VM, Daneluzzi D, et al. Antituberculosis activity of clarithromycin. Antimicrob Agents Chemother 1995; 39: 2692-2695.

40 Bosne-David S, Barros V, Verde SC, et al. Intrinsic resistance of Mycobacterium tuberculosis to clarithromycin is effectively reversed by subinhibitory concentrations of cell wall inhibitors. J Antimicrob Chemother 2000; 46: 391-395.

41 Hoffner SE, Gezelius L, Olsson-Liljequist B. In-vitro activity of fluorinated quinolones and macrolides against drug-resistant Mycobacterium tuberculosis. J Antimicrob Chemother 1997; 40: 885-888.

42 Yew WW, Piddock LJ, Li MS, et al. In-vitro activity of quinolones and macrolides against mycobacteria. J Antimicrob Chemother 1994; 34: 343-351.

43 Lu PL, Peng CF, Hwang JJ, et al. Activity of twelve second-line antimicrobial agents against Mycobacterium tuberculosis in Taiwan. J Chemother 2008; 20: 202-207.

44 Rastogi N, Labrousse V, Goh KS. In vitro activities of fourteen antimicrobial agents against drug susceptible and resistant clinical isolates of Mycobacterium tuberculosis and comparative intracellular activities against the virulent H37Rv strain in human macrophages. Curr Microbiol 1996; 33: 167-175.

45 Rastogi N, Goh KS, Ruiz P, et al. In vitro activity of roxithromycin against the Mycobacterium tuberculosis complex. Antimicrob Agents Chemother 1995; 39: 1162-1165.

46 Umubyeyi A, Rigouts L, Shamputa IC, et al. Low levels of second-line drug resistance among multidrug-resistant Mycobacterium tuberculosis isolates from Rwanda. Int J Infect Dis 2008; 12: 152-156.

47 Falzari K, Zhu Z, Pan D, et al. In vitro and in vivo activities of macrolide derivatives against Mycobacterium tuberculosis. Antimicrob Agents Chemother 2005; 49: 1447-1454.

48 Sato K, Tomioka H, Akaki T, et al. Antimicrobial activities of levofloxacin, clarithromycin, and KRM-1648 against Mycobacterium tuberculosis and Mycobacterium avium complex replicating within Mono Mac 6 human macrophage and A-549 type II alveolar cell lines. Int J Antimicrob Agents 2000; 16: 25-29.

49 Tomioka H, Sato K, Sano C, et al. Intramacrophage passage of Mycobacterium tuberculosis and M. avium complex alters the drug susceptibilities of the organisms as determined by intracellular susceptibility testing using macrophages and type II alveolar epithelial cells. Antimicrob Agents Chemother 2002; 46: 519-521.

50 Abate G, Miörner H, Ahmed O, et al. Drug resistance in Mycobacterium tuberculosis strains isolated from re-treatment cases of pulmonary tuberculosis in Ethiopia: susceptibility to first-line and alternative drugs. Int $J$ Tuberc Lung Dis 1998; 2: 580-584.

51 Chan ED, Laurel V, Strand MJ, et al. Treatment and outcome analysis of 205 patients with multidrug-resistant tuberculosis. Am J Respir Crit Care Med 2004; 169: 1103-1109.

52 Escudero E, Peña JM, Alvarez-Sala R, et al. Multidrug-resistant tuberculosis without HIV infection: success with individualised therapy. Int J Tuberc Lung Dis 2006; 10: 409-414.

53 Ferrara G, Richeldi L, Bugiani M, et al. Management of multidrug-resistant tuberculosis in Italy. Int J Tuberc Lung Dis 2005; 9: 507-513.

54 Drobniewski F, Eltringham I, Graham C, et al. A national study of clinical and laboratory factors affecting the survival of patients with multiple drug resistant tuberculosis in the UK. Thorax 2002; 57: 810-816.

55 Morcillo N, Di Giulio B, Testani B, et al. A microplate indicator-based method for determining the susceptibility of multidrug-resistant Mycobacterium tuberculosis to antimicrobial agents. Int J Tuberc Lung Dis 2004; 8: 253-259.

56 Abubakar I, Moore J, Drobniewski F, et al. Extensively drug-resistant tuberculosis in the UK: 1995 to 2007. Thorax 2009; 64: 512-515.

57 Bolhuis MS, van der Laan T, Kosterink JG, et al. In vitro synergy between linezolid and clarithromycin against Mycobacterium tuberculosis. Eur Respir J 2014; 44: 808-811.

58 Ramón-García S, Ng C, Anderson H, et al. Synergistic drug combinations for tuberculosis therapy identified by a novel high-throughput screen. Antimicrob Agents Chemother 2011; 55: 3861-3869.

59 Klemens SP, DeStefano MS, Cynamon MH. Therapy of multidrug-resistant tuberculosis: lessons from studies with mice. Antimicrob Agents Chemother 1993; 37: 2344-2347.

60 Truffot-Pernot C, Lounis N, Grosset JH, et al. Clarithromycin is inactive against Mycobacterium tuberculosis. Antimicrob Agents Chemother 1995; 39: 2827-2828.

61 Bolhuis MS, van Altena R, van Soolingen D, et al. Clarithromycin increases linezolid exposure in multidrug-resistant tuberculosis patients. Eur Respir J 2013; 42: 1614-1621.

62 Seung KJ, Becerra MC, Atwood SS, et al. Salvage therapy for multidrug-resistant tuberculosis. Clin Microbiol Infect 2014; 20: 441-446. 
63 Ahuja SD, Ashkin D, Avendano M, et al. Multidrug resistant pulmonary tuberculosis treatment regimens and patient outcomes: an individual patient data meta-analysis of 9,153 patients. PLoS Med 2012; 9: e1001300.

64 Shean KP, Willcox PA, Siwendu SN, et al. Treatment outcome and follow-up of multidrug-resistant tuberculosis patients, West Coast/Winelands, South Africa, 1992-2002. Int J Tuberc Lung Dis 2008; 12: 1182-1189.

65 Nathanson E, Lambregts-van Weezenbeek C, Rich ML, et al. Multidrug-resistant tuberculosis management in resource-limited settings. Emerg Infect Dis 2006; 12: 1389-1397.

66 Kim HR, Hwang SS, Kim HJ, et al. Impact of extensive drug resistance on treatment outcomes in non-HIV-infected patients with multidrug-resistant tuberculosis. Clin Infect Dis 2007; 45: 1290-1295.

67 Hutchison DC, Drobniewski FA, Milburn HJ. Management of multiple drug-resistant tuberculosis. Respir Med 2003; 97: 65-70.

68 Carroll MW, Lee M, Cai Y, et al. Frequency of adverse reactions to first- and second-line anti-tuberculosis chemotherapy in a Korean cohort. Int J Tuberc Lung Dis 2012; 16: 961-966.

69 Törün T, Güngör G, Ozmen I, et al. Side effects associated with the treatment of multidrug-resistant tuberculosis. Int J Tuberc Lung Dis 2005; 9: 1373-1377.

70 Williams B, Ramroop S, Shah P, et al. Multidrug-resistant tuberculosis in UK children: presentation, management and outcome. Eur Respir J 2013; 41: 1456-1458.

71 Mitnick CD, Shin SS, Seung KJ, et al. Comprehensive treatment of extensively drug-resistant tuberculosis. N Engl J Med 2008; 359: 563-574.

72 Shean K, Streicher E, Pieterson E, et al. Drug-associated adverse events and their relationship with outcomes in patients receiving treatment for extensively drug-resistant tuberculosis in South Africa. PLoS One 2013; 8: e63057.

73 Mor N, Esfandiari A. Synergistic activities of clarithromycin and pyrazinamide against Mycobacterium tuberculosis in human macrophages. Antimicrob Agents Chemother 1997; 41: 2035-2036.

74 Patel KB, Xuan D, Tessier PR, et al. Comparison of bronchopulmonary pharmacokinetics of clarithromycin and azithromycin. Antimicrob Agents Chemother 1996; 40: 2375-2379.

75 Conte JE Jr, Golden JA, Duncan S, et al. Intrapulmonary pharmacokinetics of clarithromycin and of erythromycin. Antimicrob Agents Chemother 1995; 39: 334-338.

76 Rodvold KA, Gotfried MH, Danziger LH, et al. Intrapulmonary steady-state concentrations of clarithromycin and azithromycin in healthy adult volunteers. Antimicrob Agents Chemother 1997; 41: 1399-1402.

77 Mor N, Vanderkolk J, Heifets L. Accumulation of clarithromycin in macrophages infected with Mycobacterium avium. Pharmacotherapy 1994; 14: 100-104.

78 Ishiguro $\mathrm{M}$, Koga $\mathrm{H}$, Kohno $\mathrm{S}$, et al. Penetration of macrolides into human polymorphonuclear leucocytes. J Antimicrob Chemother 1989; 24: 719-729.

79 Jia L, Tomaszewski JE, Hanrahan C, et al. Pharmacodynamics and pharmacokinetics of SQ109, a new diamine-based antitubercular drug. Br J Pharmacol 2005; 144: 80-87.

80 Sotgiu G, Centis R, D'Ambrosio L, et al. Efficacy, safety and tolerability of linezolid containing regimens in treating MDR-TB and XDR-TB: systematic review and meta-analysis. Eur Respir J 2012; 40: 1430-1442.

81 Fortún J, Martín-Dávila P, Navas E, et al. Linezolid for the treatment of multidrug-resistant tuberculosis J Antimicrob Chemother 2005; 56: 180-185.

82 Alffenaar JW, van der Laan T, Simons S, et al. Susceptibility of clinical Mycobacterium tuberculosis isolates to a potentially less toxic derivate of linezolid, PNU-100480. Antimicrob Agents Chemother 2011; 55: 1287-1289.

83 Balasubramanian V, Solapure S, Iyer H, et al. Bactericidal activity and mechanism of action of AZD5847, a novel oxazolidinone for treatment of tuberculosis. Antimicrob Agents Chemother 2014; 58: 495-502.

84 Stover CK, Warrener P, VanDevanter DR, et al. A small-molecule nitroimidazopyran drug candidate for the treatment of tuberculosis. Nature 2000; 405: 962-966.

85 Matsumoto M, Hashizume H, Tomishige T, et al. OPC-67683, a nitro-dihydro-imidazooxazole derivative with promising action against tuberculosis in vitro and in mice. PLoS Med 2006; 3: e466.

86 Kannan K, Vázquez-Laslop N, Mankin AS. Selective protein synthesis by ribosomes with a drug-obstructed exit tunnel. Cell 2012; 151: 508-520.

87 Krokidis MG, Márquez V, Wilson DN, et al. Insights into the mode of action of novel fluoroketolides, potent inhibitors of bacterial protein synthesis. Antimicrob Agents Chemother 2014; 58: 472-480.

88 Champney WS, Tober CL. Structure-activity relationships for six ketolide antibiotics. Curr Microbiol 2001; 42: 203-210.

89 U.S. Food and Drug Administration. Telithromycin (marketed as Ketek) Information. www.fda.gov/drugs/ drugsafety/postmarketdrugsafetyinformationforpatientsandproviders/ucm107824.htm Date last accessed: September 14, 2014. Date last updated: January 19, 2010.

90 Clay KD, Hanson JS, Pope SD, et al. Brief communication: severe hepatotoxicity of telithromycin: three case reports and literature review. Ann Intern Med 2006; 144: 415-420.

91 Brown SD. Benefit-risk assessment of telithromycin in the treatment of community-acquired pneumonia. Drug Saf 2008; 31: 561-575.

92 Volberg WA, Koci BJ, Su W, et al. Blockade of human cardiac potassium channel human ether-a-go-go-related gene (HERG) by macrolide antibiotics. J Pharmacol Exp Ther 2002; 302: 320-327.

93 Simkó J, Csilek A, Karászi J, et al. Proarrhythmic potential of antimicrobial agents. Infection 2008; 36: 194-206.

94 Pranger $\mathrm{AD}$, van Altena R, Aarnoutse RE, et al. Evaluation of moxifloxacin for the treatment of tuberculosis: 3 years of experience. Eur Respir J 2011; 38: 888-894.

95 Gler MT, Skripconoka V, Sanchez-Garavito E, et al. Delamanid for multidrug-resistant pulmonary tuberculosis. N Engl J Med 2012; 366: 2151-2160.

96 Worley MV, Estrada SJ. Bedaquiline: a novel antitubercular agent for the treatment of multidrug-resistant tuberculosis. Pharmacotherapy 2014; 34: 1187-1197. 